\title{
NEW CAPABILITIES OF COORDINATE DETECTORS ON THE BASIS OF
} STRAWS

\section{V.D. Peshekhonov ${ }^{1}$}

Joint Institute for Nuclear Research (JINR)

Dubna, Russia

E-mail: Vladimir.Peshekhonov@cern.ch

\section{Abstract}

Two prototypes based on segmented and high pressure straws had been developed and tested. Efficiency, spatial resolution and other parameters had been studied. 
The need in reliable, relatively cheap and low-mass budget tracking detectors with good spatial resolution which, in addition, allow for continuous refreshing of the working substance ionized by charged particles justifies an interest in gas filled detectors. The factors limiting the use of straw detectors include high occupancy and insufficiently high spatial resolution. In order to make the limitations less stringent the R@D with granulated straws and with straws filled with a gas under high pressure has been fulfilled which opens a possibility to achieve a good granularity and a spatial resolution comparable with other detectors.

A double layer granulated straw prototype (GSP) based on 96 granulated straws $4 \mathrm{~mm}$ in diameter and $410 \mathrm{~mm}$ long has been developed for the beam tests [1]. Thinfilm tubes similar to the ATLAS TRT tubes have been employed [2]. The prototype has 360 registration channels (segments). A readout and anode voltage supply are accomplished by the custom-developed low-mass transmission lines [3, 4]. Each straw contains the multisegmented anode with a set of wires consecutively integrated into one element by glass capillary joints and low-mass spacers with two contact wires being used for leading out of the straw and connection with the transmission line buses.

The present study of the high pressure prototype (HPP) has demonstrated that straw detectors can operate under a gas pressure up to 5 bar with a negligible gas leak. The observed changes in the straw size due to the increased gas filling pressure have been reduced substantially for the reinforced straws by employing the longitudinal carbon thin strips glued onto their surface. The developed two layer HPP employs the reinforced straws with the inner diameter of $9.53 \mathrm{~mm}$. The straw tubes are similar to the unreinforced tubes of the COMPASS straw tracker [5]. The diameter of anode wire used in both prototypes is $30 \mu \mathrm{m}$.

The two prototypes have been tested in the SPS test beam at CERN. The prototypes have been placed centrally in the pixel telescope EUDET [6] which has the reconstruction accuracy of $\sim 3 \mu \mathrm{m}$ near the center. The output signals from the prototypes arrived to the DAQ and then were transferred to EUDET DAQ. Both prototypes were blown with the gas mixture $\mathrm{Ar} / \mathrm{CO} 2$ (80/20) under pressure of 1 bar for the GSP and under pressure varied from 1 to 5 bar for the HPP. The discriminator thresholds were about 3 and $4.5 \mathrm{fC}$, respectively.

The main tasks of the beam test for the GSP were determination of the efficiency, spatial resolution and insensitive range of the straws in the region of spacers [7]. The efficiency of the straws was about $\sim 98 \%$ and the spatial resolution was $\sim 180$ $\mu \mathrm{m}$. For the measurements performed, the gas gain was about $6 \times 10^{4}$. The length of ineffective region along the straw axis for the spacer zone is less than $7 \mathrm{~mm}$. So, the insensitive region due to straw internal elements is less than $5 \%$ of full sensitive area of the prototype with the granularity of $4 \mathrm{~cm}^{2}$. The area in which deterioration of the spatial resolution near these elements takes place makes not more than $1.0 \%$ of the detector sensitive area.

The main task of the HPP beam test was investigation of a spatial resolution as a function of the gas mixture pressure [8]. Figure 1 illustrates the distribution of the residuals between the track of the EUDET pixel detectors. The r.m.s. width of narrow Gaussian peak of the straw is $32 \mu \mathrm{m}$. The spatial resolution uniformly averaged over the distance between a track and the anode wire is $49 \mu \mathrm{m}$ and $170 \mu \mathrm{m}$ under the gas pressure of 3 and 1 bar, respectively (Fig.1, right panel). The averaged spatial hit 
resolution for the nearest to anode interval ( $1 \mathrm{~mm}$ long) and for the distant part of the straw radius is $\sim 95(330)$ and $\sim 37(130) \mu \mathrm{m}$, respectively, for the straw under the pressure of 3(1) bar.


Fig. 1. Left: The spatial resolution ( $\sigma$ ) of the HPP straw, $\mathrm{HV}$ is $3.05 \mathrm{kV}$ and pressure $-3 \mathrm{bar}$.

Right: The spatial resolution along the straw radius for the pressure -1 bar and gas gain - $6 \times 10^{4}$ (top curve) and 3 bar (bottom curve), HV was 3.05 and , the efficiency were $99 \%$ for the both measurements.

The beam test has demonstrated the feasibility of building large size detectors based on the segmented straws and/or with the gas mixture pressure in the range of 1 3 bar. The granularity of $4 \mathrm{~mm}$ in diameter straws may vary from 1 to $30 \mathrm{~cm}^{2}$ and their length can be up to $4 \mathrm{~m}$, and material budget of the detector is smaller in comparison with other detectors. The spatial resolution of the detector with gas under pressure 3 bar can be better than $50 \mu \mathrm{m}$. The bench comparison testing of rate capability had been fulfilled for one straw of the GSP and had shown that rate capability is retained.

\section{References.}

[1]. Yu. V. Gusakov et al., Multichannel Prototype Detector Based on Segmented Straws, Phys. Part. Nucl. 7, (2010) 132.

[2]. Inner Detector Technical Design Report, CERN/LHCC/97-17, ATLAS TDR 5, 1997.

[3]. K. Davkov et al., Development of segmented straws for very high-rate capability coordinate detector, Nucl. Instr. and Meth. in Phys. Res. 584 (2008) 285.

[4]. S. E. Vasilyev et al., Signal Transmission Lines for Large-Size Segmented Straw Detectors, Instr. and Exp.Techn. 51 (2008) 820.

[5]. V.N. Bychkov et al., The large size straw drift chambers of the COMPASS experiment, Nucl. Instr. and Meth. in Phys. Res. 556 (2006) 66.

[6]. URL: http://www.eudet.org.

[7]. S.N. Bazylev et al., A Prototype Coordinate Detector Based on Granulated Thin-Walled Drift Tubes, submitted to Nucl. Instr. and Meth. in Phys. Res.

[8]. V.I. Davkov et al., Spatial Resolution of Thin-Walled Drift Tubes with High Pressure of Gas Mixture, submitted to Nucl. Instr. and Meth. in Phys. Res. 